\title{
Assessing the health effects of extreme temperature and development of adaptation strategies to climate change in selected countries in the Asia-Pacific region
}

\author{
Liangliang Cheng a and Cunrui Huang a * \\ a School of Public Health, Sun Yat-sen University, Guangzhou, China \\ * Corresponding author.Email: huangcr@mail.sysu.edu.cn
}

\begin{abstract}
While studies show that climate change is exacerbating health effects due to extreme hot weather, scientific evidence in the Asia-Pacific region remains scarce. In this study, we aim to assess the health effects of extreme temperature, identify individual and community factors contributing to population vulnerability, and develop adaptation strategies for temperature-related health risks. Various methods were adopted for different research purposes in the study. Distributed lag non-linear model and conditional Poisson model were used to assess temperature-health associations. Subgroup analysis, hierarchical Bayesian model and logistic regression model were used for identifying vulnerable subgroups. Results showed that extreme temperature is associated with a range of human morbidity or mortality outcomes in the selected Asia-Pacific localities. The interaction of extreme temperature and air pollution also increased health risks. It is projected that heat-related health effects will increase dramatically under climate change scenarios with urban expansion and ageing population in the near future. Vulnerable subgroups in the study were identified as the elderly, people with pre-existing diseases, outdoor workers, residents living in high population density areas, and those with low socio-economic status. In addition, a few cities developed adaptation strategies to manage the health risks as a result of extreme temperatures, such as heat-health action plans and early warning systems. Future efforts should be taken to develop and evaluate the effectiveness of adaptation strategies for alleviating public health impacts of climate change in the Asia-Pacific region.
\end{abstract}

\section{KEYWORDS}

Adaptation, Climate change, Health effect, Morbidity, Mortality, Temperature, Vulnerability

DOI

https://doi.org/10.30852/sb.2019.854

\section{DATES}

Received: 3 September 2019

Published (online): 4 December 2019

Published (PDF): 12 December 2019

\section{HIGHLIGHTS}

» Extreme temperatures were associated with a wide range of health outcomes in the AsiaPacific localities.

» The elderly, people with preexisting diseases, outdoor workers, residents living in high population density areas, and those with low socio-economic status were particularly sensitive to heat exposure.

» Cost-effective adaptation strategies should be developed to address the health impacts of climate change.

\section{INTRODUCTION}

A range of early impacts of climate change have been observed over recent decades, which include increasing ambient temperatures, changing precipitation patterns and an increase in the frequency of extreme weather events (Stocker, 2014). With growing concerns about global warming and deadly heatwaves, the health effects of temperature are fast becoming a global public health challenge in the 21st century (Hajat, O'Connor, \& Kosatsky, 2010; Watts et al., 2018). Health effects due to extreme temperature are the most significant source of weather-related public health problems, and also the most direct and well-understood impact of climate change on human health (Huang, Barnett, Wang, \& Tong, 2012; Kinney, 2012). The Intergovernmental Panel on Climate Change (IPCC) has stated that a future increase in temperature will lead to excess mortality and morbidity among vulnerable populations for many regions (IPCC, 2014).

To date, most previous analyses have focused on mortality/morbidity concerning heat events in high-income nations, with few studies investigating 
low- and middle-income countries (Green et al., 2019; Kjellstrom et al., 2016). Countries in the Asia-Pacific region, which is home to more than half of the world's population, and changes in the Earth's climate are clearly impacting human health and survival. Also, with unique geography and population groups, the Asia-Pacific region is exceptionally diverse, and includes least developed countries, rapidly emerging economies and developed nations. Sensitive population and spatial heterogeneity in terms of heat vulnerability may vary considerably from one environment to another. More importantly, response strategies to address the health effects of temperature have not been sufficiently considered in current public health practices and activities (Benmarhnia, Deguen, Kaufman, \& Smargiassi, 2015; Huang et al., 2013).

In this article, we aim to summarize the purpose of the study, the methodology used, and the main research findings. Cities in south China, Viet Nam and Thailand were selected as the study sites for their typically hot and humid climates. Specifically, this study attempted to quantify heat-related health effects, improve knowledge on the burden of disease attributable to extreme temperatures, examine individual and community factors contributing to population vulnerability, and provide decision-makers with adaptation strategies to extreme temperatures.

\section{DATA AND METHODOLOGY}

\subsection{Data sources}

Data on health outcomes including mortality and mobility data were gathered in this study. Various forms of data were collected, such as work injury claim and insurance data (Guangzhou, 2011-2012), ambulance dispatch data (Shenzhen, 2015-2016), stroke surveillance data (Shenzhen, 2005-2016), birth registry data (Shenzhen, 2005-2012), life expectancy data (Guangzhou, 2010-2015), medical students survey data (Nationwide, 2017) in China, hospitalization and death data (Ho Chi Minh City and the Mekong Delta Region, 2010-2013) in Viet Nam, and mortality data (Nationwide, 1999-2008) in Thailand. The majority of data were obtained from Health Administrative Departments, Centres for Disease Control, and Hospital Information Systems.

In addition, meteorological and air pollutant data such as daily temperature, relative humidity, particulate matter with an aerodynamic diameter less than $2.5 \mu \mathrm{m}$ $\left(\mathrm{PM}_{2.5}\right)$, ozone $\left(\mathrm{O}_{3}\right)$, and sulphur dioxide $\left(\mathrm{SO}_{2}\right)$ were also included. These indicators were obtained from National Climate Centers, Environmental Protection Agencies, and some Real-time Publishing Platforms (e.g., Air
Quality Real-time Publishing Platform of China). There are no universal definitions for the terms "extreme temperatures" and "heatwaves". In this study, extreme temperature was defined as daily temperatures exceeding a given threshold value, for instance, 90th/95th of the temperature range. Heatwave was defined based on the magnitude, duration, and frequency of extreme temperatures, such as a few consecutive days of temperatures above a certain threshold.

Ethics approval was granted by the Ethics Committee of Sun Yat-sen University prior to data collection. Descriptive analysis of the meteorological, air pollutant and health outcome variables in different Asia-Pacific cities can be found in the published literature by our team (Bao et al., 2019; Dang et al., 2019; He et al., 2018; Huang et al., 2018; Liao et al., 2019; Liu et al., 2019; Ma et al., 2019; Phung, Chu, Tran, \& Huang, 2018; Sheng et al., 2018; Wang et al., 2019).

\subsection{Methodology}

A variety of statistical methods were used for analysis, ranging from simple linear models to complex non-linear models. In general, ample statistical methods were developed in the field of environmental epidemiology, and the methods used varied for different research purposes.

When assessing the health effects of extreme temperatures, distributed non-linear lag model (DLNM) with an ecological design or conditional Poisson model with a time-stratified case-crossover design are frequently used (Dang et al., 2019; Huang et al., 2018; Sheng et al., 2018). In these models, different mortality and morbidity outcomes (e.g., daily counts of death or ambulance dispatches) were set as dependent variables; environmental exposure indicators (e.g., daily mean temperature or air pollution) were independent variables; and other time-invariant confounders (e.g., socio-demographic characteristics) and time-varying confounders (e.g., long-time trend and seasonality) were also controlled. To identify heat vulnerability such as population characteristics or spatial heterogeneity, subgroup analysis, hierarchical Bayesian model with an ecological design or logistic regression model with a case-only design were adopted in the study (Bao et al., 2019; He et al., 2018; Ma et al., 2019; Phung et al., 2018). All statistical analyses were performed using R software (version 3.4.0).

\section{RESULTS AND DISCUSSION}

\subsection{Heat-related health effects and attributable burdens}

A positive temperature-health association was found among different human morbidity outcomes. 

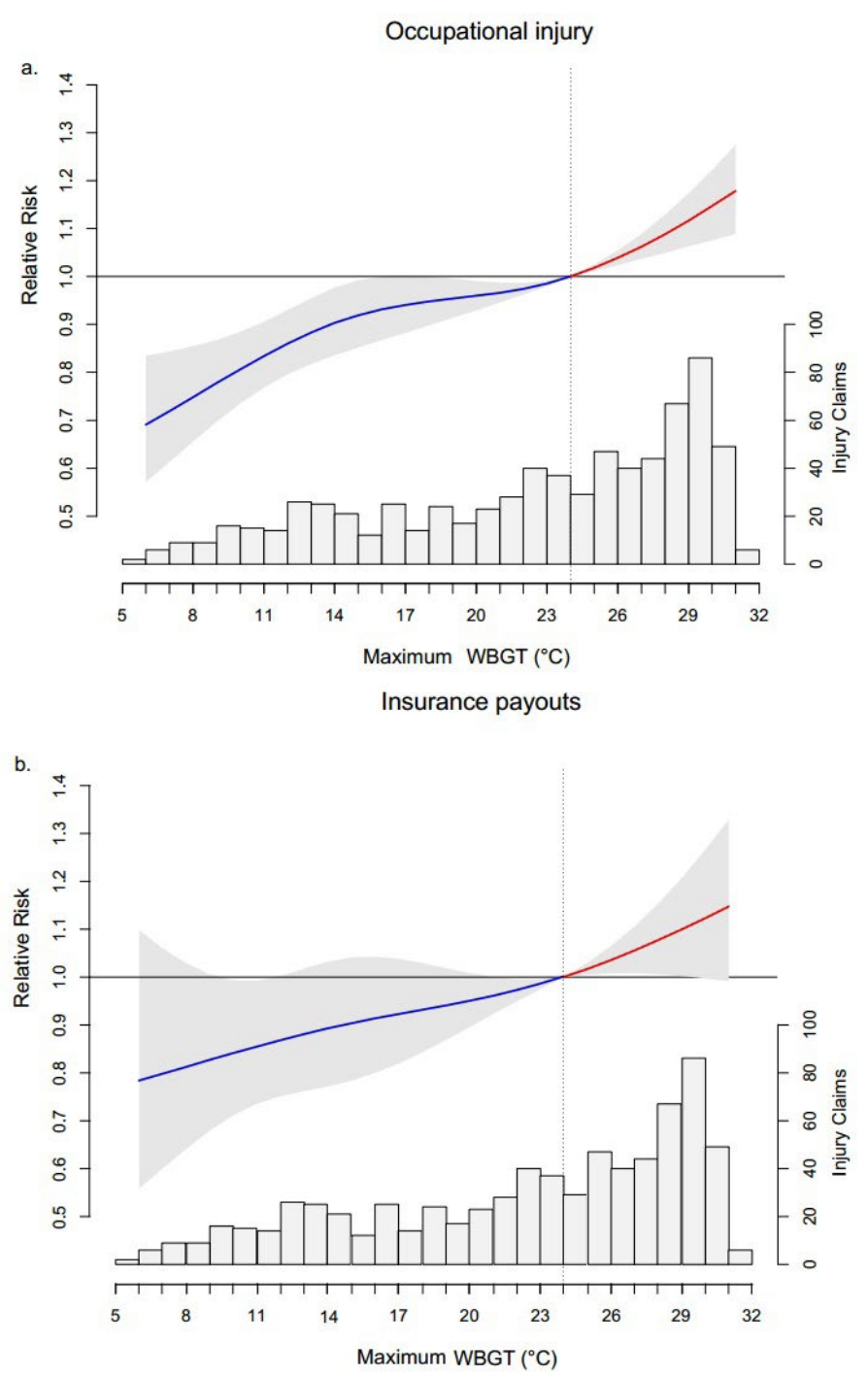

FIGURE 1. The exposure-response relationship between maximum WBGT and occupational injury (a) and insurance payouts (b) in Guangzhou, China.
Risk of work-related injuries increased by $15 \%(\mathrm{RR}=1.15$, 95\%CI: 1.08-1.22) when wet bulb globe temperature (WBGT) reached $30{ }^{\circ} \mathrm{C}$, with $4.8 \%$ of injuries and $4.1 \%$ of related insurance payouts attributing to extreme temperatures in Guangzhou, China (Figure 1). Ambulance dispatches during extremely hot days increased by $19 \%$ ( $\mathrm{RR}=1.19,95 \% \mathrm{CI}$ : 1.11-1.27) in comparison with the optimum temperature of $22{ }^{\circ} \mathrm{C}$ in Shenzhen, another most populous city of southern China. In Viet Nam, the Mekong Delta showed 6.1\% (95\%CI: 5.9-6.2) increase of hospital admissions when average temperatures rose by $5{ }^{\circ} \mathrm{C}$. Besides, the interaction between temperature and air pollutants may further aggravate the situation. For instance, the risk of $\mathrm{PM}_{10}$ increased by $90 \%$ ( $95 \% \mathrm{CI}$ : 19-205) among live births of small for gestational age (SGA) when exposed to extreme temperatures in Shenzhen, China.

In addition, human mortality was also associated with extreme temperatures. Research conducted in Thailand, based on death data from 1999 to 2008, suggested that a higher intensity level of heatwaves resulted in more deaths ( $\mathrm{RR}=1.126,95 \% \mathrm{CI}: 1.069-1.186$ ) (Figure 2). A similar effect on mortality was also found in Viet Nam. Heatwaves significantly increased the risk of death of cause-specific diseases (e.g., respiratory disease, RR:1.45, 95\%CI: 1.25-1.70). Besides, future climate change coinciding with rapid population growth or ageing may also present a big challenge in regard to premature deaths. The annual heat-related years of life lost (YLLS) in Guangzhou, China under the RCP8.5 scenarios in the 2030s, 2060s, and 2090s will increase to 2.2, 7.0 and 11.4 thousand, respectively (Liu et al., 2019).
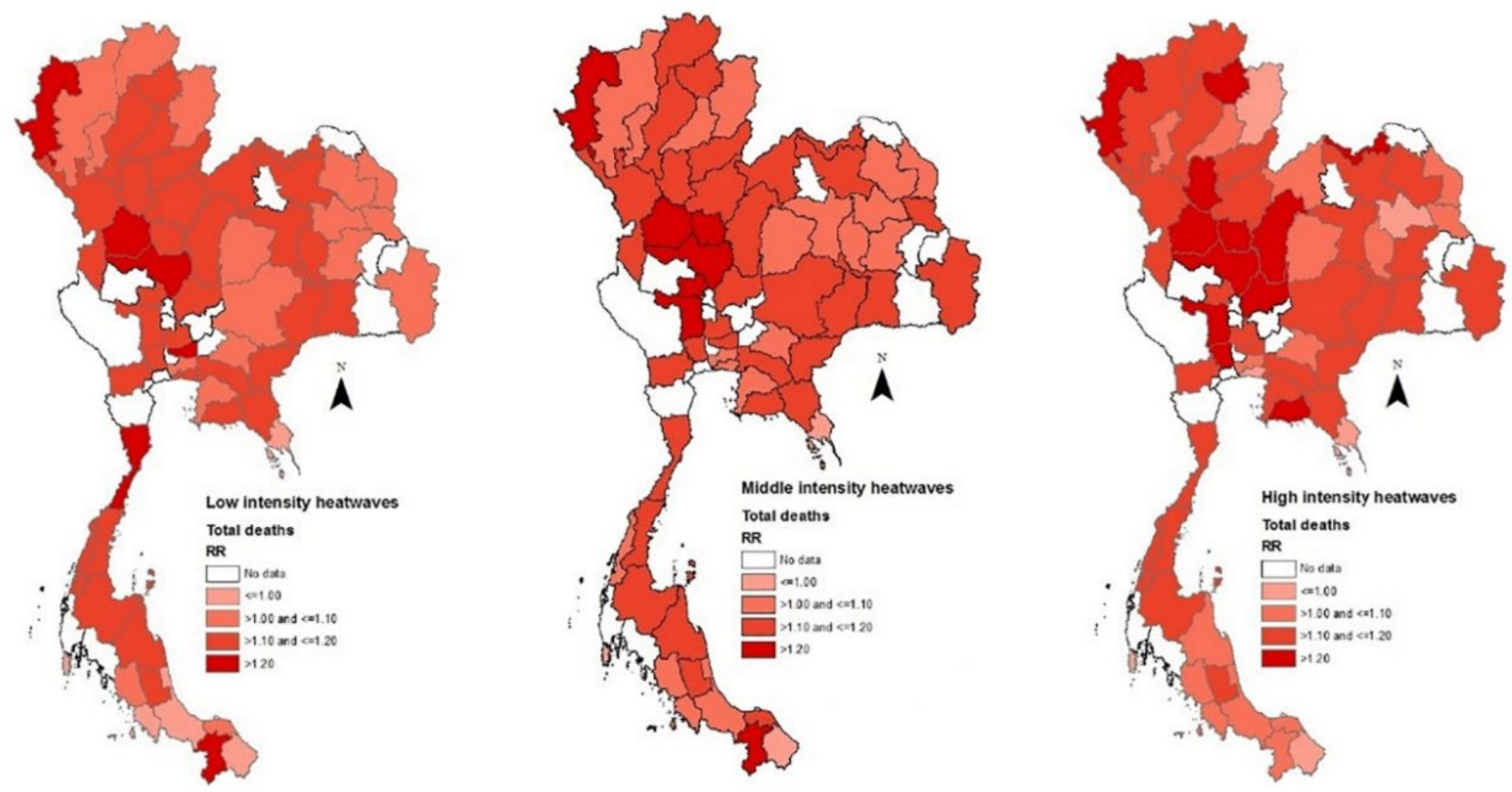

FIGURE 2. Heatwave impacts on total deaths in 60 provinces in Thailand. 
These studies reveal that extremely high temperatures increase the risk of different mortality and morbidity outcomes in cities of the Asia-Pacific region. The results are consistent with findings reported in many previous studies around the world. The extreme temperatures are associated with a cumulative $7.5 \%$ and $5.1 \%$ higher rate of all-cause emergency department visits and deaths from 2005 to 2012 in England (Wellenius et al., 2017), and heat-related mortality increased by $146 \%$ from 1960 to 2009 in India (Mazdiyasni et al., 2017). A recent systematic review also reported that $93 \%$ of studies found positive associations between heat and morbidity/mortality rates in low- and middle-income countries (Green et al., 2019). Further, since high temperatures accelerate the generation and spread of air pollutants, and also influence the efficacy of toxic substances entering the body, it is biologically plausible that ambient temperature and air pollution can interact to affect health outcomes (Csavina et al., 2014; Gordon, Johnstone, \& Aydin, 2014). A recent study in Australia has found strengthened effects of $\mathrm{PM}_{2.5}$ exposure in low ambient temperatures on low birth weight (Chen, Guo, Abramson, Williams, \& Li, 2018). Also, future climate change coinciding with rapid urban expansion or population ageing may exacerbate health risks in the Asia-Pacific region. This finding was consistent with other projection studies; for instance, the annual additional deaths due to climate change would increase to approximately 250,000 between 2030 and 2050 globally (World Health Organization [WHO], 2014). It has been demonstrated that rapid population growth and demographic change, especially in urban areas, could lead to land use/cover change, air pollution, overcrowding, changes in physical activity patterns, and inadequate service capacity for sanitation. All of them could elevate the human risk of climate change in the future (Li et al., 2016).

\subsection{Population vulnerability to extreme temperatures}

Temperature-related health effects can be modified by different characteristics of a vulnerable population. In this study, multiple vulnerable subgroups were identified, consisting mainly of the elderly, people with pre-existing diseases, outdoor workers, residents living in high population density areas, and those with low socio-economic status.

Different sensitivity to heat was associated with different individual characteristics. The elderly $(\mathrm{RR}=1.28$, 95\%CI: 1.14-1.47) or people with pre-existing respiratory diseases ( $\mathrm{RR}=1.30,95 \% \mathrm{CI}: 1.19-1.42)$ were more likely to be hospitalized, and also more likely to die when compared with the optimum temperature in Ho Chi

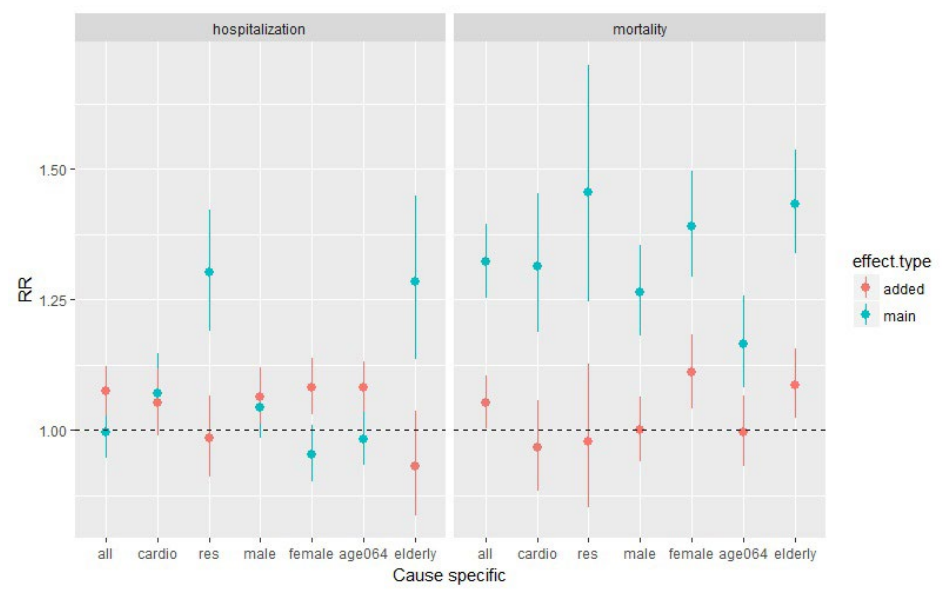

FIGURE 3. Vulnerable subgroups of hospitalization and mortality during hot days in Ho Chi Minh City, Viet Nam. Main effects: compare with the optimum value of temperature. Added effects: compare with nonheatwave days. The left panel is for hospitalization, and the right panel is for mortality. Cardio: cardiovascular disease. Res: respiratory disease.

Minh City of Viet Nam (Figure 3). A significant association between ambulance dispatches and extremely high temperatures was identified among the population over 60 years old in Shenzhen, China (RR=1.27, 95\%CI: 1.041.28). The occupational population in the transport and construction sectors, mainly working outdoors, have an increased injury risk of 50\% (RR=1.50, 95\%CI: 1.4261.580) during hot days in Guangzhou, China. Workers with low educational attainment showed a high injury risk with increasing WBGT (RR=1.19, 95\% CI:1.08-1.32).

In addition, spatial heterogeneity in terms of heat vulnerability was also observed. In the Mekong Delta Region of Viet Nam, the district-level risks ranged from $24.4 \%$ to $55.2 \%$ in admissions per $5{ }^{\circ} \mathrm{C}$ increase in average temperature. Temperature-hospitalization risk increased by $1.3 \%$ (95\%CI: 1.2-1.4) for each increase of 1,000 persons $/ \mathrm{km}^{2}$ in population density. In contrast, the risk decreased up to $6.8 \%$ (95\%CI: 6.6-6.9) in those districts with many rural populations. In Thailand, northern and central regions were more vulnerable to heatwaves, and the proportion of the elderly population was the major driver behind the spatial heterogeneity (Figure 2).

Vulnerability can be explained by some possible mechanisms as discussed in the published literature of this study, although the casual effect is not well understood. The older population are vulnerable mainly due to the degeneration of their thermoregulatory systems, the increase of co-morbidities, as well as medication use (Bao et al., 2019; Dang et al., 2019; Huang et al., 2018). Outdoor workers, more likely to be exposed to external thermal environments and produce intra-body heat while engaged in heavy workloads, are also vulnerable to hot weather (Ma et al., 2019; Sheng et al., 2018). The condition of cardiovascular endocrine, urinary and 
respiratory systems will influence the rate of body heat dissipation. Therefore, people with specific diseases that compromise body cooling mechanisms are more susceptible to heat (Dang et al., 2019). People with low educational attainment or income may have less opportunity to respond to heat warnings, low prevalence of air conditioning, lack of medical care, and health insurance shortage (Ma et al., 2019). With rapid urbanization and population migration, high-density cities and towns are hotter than surrounding rural areas due to a lack of green space and reduction of airflow around highly crowded buildings (Liu et al., 2019; Phung et al., 2018). Residents in high-density areas may face vital health challenges on mortality and morbidity.

\subsection{Development of adaptation strategies to climate change}

It is suggested that the development of adaptation strategies needs to be better planned and should take into account differences in population sensitivity and future drivers (e.g., climate change, rapid urbanization and population ageing). Considering these challenges, we identified several relevant policies and measures to address the health impacts of heatwaves and climate change in the region.

The differences in sensitivity to heat would play an essential role in developing any response strategy. For instance, considering the significant spatial variation of heat vulnerability in the Mekong Delta Region, our researchers suggested that public health intervention measures should be developed using the city/ district scale rather than the province scale (Phung et al., 2018). In addition, evidence also reveals that the rapid urbanization and population ageing would significantly aggravate adverse heat-related effects under all climate change scenarios. The assessment of future health risks could aid in improving the design of public health interventions and policies, preparedness of adaptation strategies, and healthcare planning (Liu et al., 2019). Furthermore, adaptation strategies may fall into autonomous and planned actions. Although autonomous adaptation can occur without coordinated scheming in individual or community levels and are usually reactive by nature, well-planned adaptation will involve deliberate policy actions with conscious intervention based on anticipated risks (Huang et al., 2013; Huang et al., 2011). Thus, planning is more critical for public health communities to cope with the adverse effects of heatwaves.

Several adaptation strategies were proposed in this study. Heat-health action plans (HAPs) could help decrease adverse health impacts of hot weather (Chau, Chan, \& Woo, 2009; Tan et al., 2007). For instance, in 2012 the Chinese state government released The Administrative Measures on Heatstroke Prevention (AMHP2012) to address intensive heat events. Some critical countermeasures, including applying new materials and technologies, constructing protective equipment, adjusting shift time, and paying high-temperature subsidies to workers during hot days. Besides, heat-health warning systems (HHWSs) can be developed to cope with impending hazardous hot weather (Hajat et al., 2010). Figure 4 shows the typical process within an HHWS, involving weather forecasting, trigger of the system, risk communication and action recommendation (World Meteorological Organization [WMO] \& WHO, 2015). The benchmark for issuing a warning varies from place to place, based on different local health responses to extreme weather. Beyond the threshold point, information is disseminated to various stakeholders so specific interventions can be taken, accordingly. For instance, HHWSs have been set up in several cities such as Shanghai, Nanjing and Harbin in China. A series of operations, such as risk dissemination through various media, health education, mobilization of medical and public services, maintenance of water and cooling facilities, are performed by the health departments, collaborating with other supporting agencies. However, no study has systematically evaluated the effectiveness and economic aspects of HHWSs among these cities.

Although a few Asia-Pacific countries have developed public health adaptation strategies to climate change, the coverage and diversity are far from sufficient. Strategies like urban planning for the cool city, vulnerability

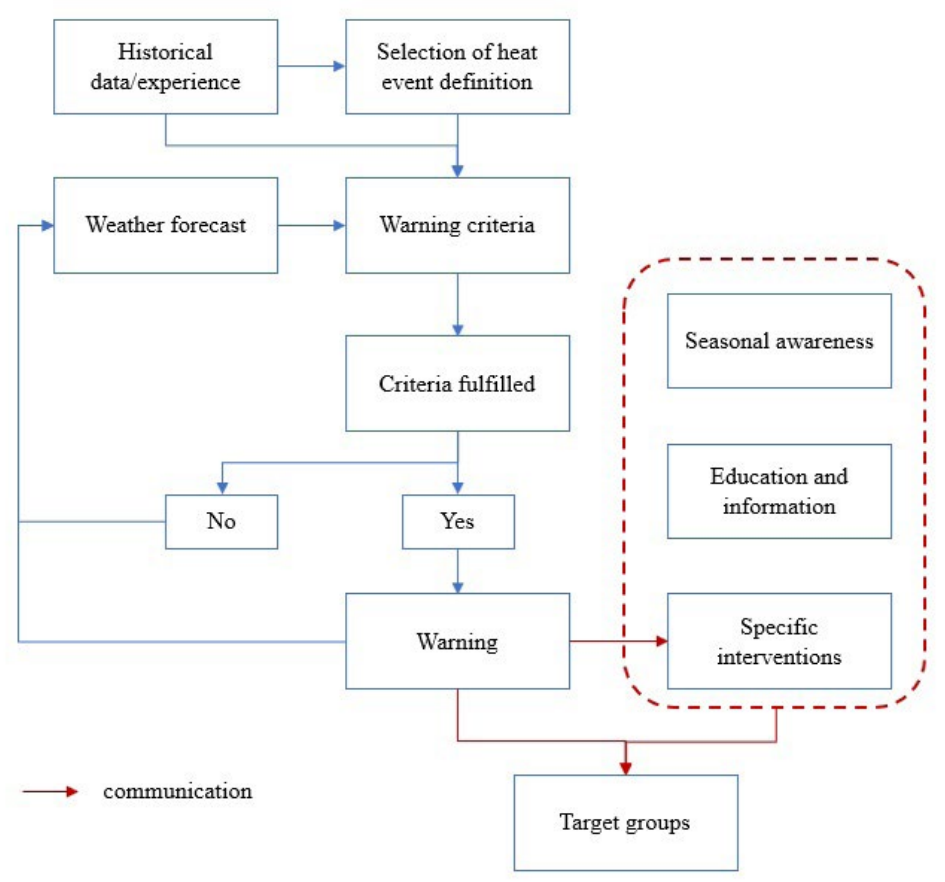

FIGURE 4. Flow diagram displaying the operation of heat-health warning systems (red box includes key elements of action plan after issuing a warning). 
mapping, and real-time surveillance systems were only reported in high-income countries (Stone, Hess, \& Frumkin, 2010; Wolf \& McGregor, 2013). Besides that, the literature on how the strategy is implemented and whether it has a protecting effect on health, is very limited. In addition, a survey conducted in China showed approximately $90 \%$ of medical students embraced their role in tackling climate change, but half of them reported themselves and the health sectors were not adequately prepared (Liao et al., 2019). Therefore, multi-city analyses and further exploration of susceptible diseases in the Asia-Pacific region are needed to generate more evidence on heat-related health effects. Adaptation strategies for alleviating public health impacts of climate change should be developed in a timely manner as well as systematically evaluated. In particular, educational efforts should reinforce eco-medical literacy development and capacity building in the era of climate change.

\section{CONCLUSIONS}

We endeavoured to investigate the health effects of extreme temperature, identify factors contributing to population vulnerability and formulate local adaptation strategies for heat-related health risks. In this study, we provide sound evidence that extreme temperatures are associated with a wide range of human health issues in the Asia-Pacific region and, finally, significant individual- and community-level modifications were explored, and future research directions and policy suggestions were also proposed. The study will have the potential to make a significant contribution to protecting the health of present and future generations in the Asia-Pacific region.

\section{ACKNOWLEDGEMENT}

This study was funded by the Asia-Pacific Network for Global Change Research (CRRP2016-10MY-Huang).

\section{REFERENCES}

Bao, J., Guo, Y., Wang, Q., He, Y., Ma, R., Hua, J., ... \& Huang, C. (2019). Effects of heat on first-ever strokes and the effect modification of atmospheric pressure: A time-series study in Shenzhen, China. Science of The Total Environment, 654, 1372-1378.

Benmarhnia, T., Deguen, S., Kaufman, J. S., \& Smargiassi, A. (2015). Vulnerability to heat-related mortality. Epidemiology, 26(6), 781-793.

Chau, P. H., Chan, K. C., \& Woo, J. (2009). Hot weather warning might help to reduce elderly mortality in Hong Kong. International Journal of Biometeorology, 53(5), 461.

Chen, G., Guo, Y., Abramson, M. J., Williams, G., \&
Li, S. (2018). Exposure to low concentrations of air pollutants and adverse birth outcomes in Brisbane, Australia, 2003-2013. Science of the Total Environment, 622, 721-726.

Csavina, J., Field, J., Félix, O., Corral-Avitia, A. Y., Sáez, A. E., \& Betterton, E. A. (2014). Effect of wind speed and relative humidity on atmospheric dust concentrations in semi-arid climates. Science of the Total Environment, 487, 82-90.

Dang, T. N., Honda, Y., Van Do, D., Pham, A. L. T., Chu, C., Huang, C., \& Phung, D. (2019). Effects of extreme temperatures on mortality and hospitalization in Ho Chi Minh City, Vietnam. International Journal of Environmental Research and Public Health, 16(3), 432.

Gordon, C. J., Johnstone, A. F., \& Aydin, C. (2011). Thermal stress and toxicity. Comprehensive Physiology, 4(3), 995-1016.

Green, H., Bailey, J., Schwarz, L., Vanos, J., Ebi, K., \& Benmarhnia, T. (2019). Impact of heat on mortality and morbidity in low and middle income countries: A review of the epidemiological evidence and considerations for future research. Environmental Research. 171, 80-91.

Hajat, S., O'Connor, M., \& Kosatsky, T. (2010). Health effects of hot weather: From awareness of risk factors to effective health protection. The Lancet, 375(9717), 856-863.

Hajat, S., Sheridan, S. C., Allen, M. J., Pascal, M., Laaidi, K., Yagouti, A., ... \& Kosatsky, T. (2010). Heat-health warning systems: a comparison of the predictive capacity of different approaches to identifying dangerously hot days. American Journal of Public Health, 10o(6), 1137-1144.

He, Y., Zhang, X., Ren, M., Bao, J., Huang, C., Hajat, S., \& Barnett, A. (2018). Assessing effect modification of excess winter death by causes of death and individual characteristics in Zhejiang Province, China: A multi-community case-only analysis. International Journal of Environmental Research and Public Health, 15(8), 1663.

Huang, C., Barnett, A. G., Xu, Z., Chu, C., Wang, X., Turner, L. R., \& Tong, S. (2013). Managing the health effects of temperature in response to climate change: challenges ahead. Environmental Health Perspectives, 121(4), 415-419.

Huang, C., Cheng, J., Phung, D., Tawatsupa, B., Hu, W., \& Xu, Z. (2018). Mortality burden attributable to heatwaves in Thailand: A systematic assessment incorporating evidence-based lag structure. Environment International, 121, 41-50.

Huang, C., Vaneckova, P., Wang, X., FitzGerald, G., Guo, Y., \& Tong, S. (2011). Constraints and barriers to public health adaptation to climate change: A review 
of the literature. American Journal of Preventive Medicine, 40(2), 183-190.

Huang, C., Barnett, A. G., Wang, X., \& Tong, S. (2012). The impact of temperature on years of life lost in Brisbane, Australia. Nature Climate Change, 2(4), 265.

Intergovernmental Panel on Climate Change, (2014). Climate Change 2014 - Impacts, Adaptation and Vulnerability: Regional Aspects. Cambridge University Press.

Kinney, P. L. (2012). Health: A new measure of health effects. Nature Climate Change, 2(4), 233.

Kjellstrom, T., Briggs, D., Freyberg, C., Lemke, B., Otto, M., \& Hyatt, O. (2016). Heat, human performance, and occupational health: a key issue for the assessment of global climate change impacts. Annual Review of Public Health, 37, 97-112.

Li, X., Song, J., Lin, T., Dixon, J., Zhang, G., \& Ye, H. (2016). Urbanization and health in China, thinking at the national, local and individual levels. Environmental Health, 15(1), 32.

Liao, W., Yang, L., Zhong, S., Hess, J. J., Wang, Q., Bao, J., \& Huang, C. (2019). Preparing the next generation of health professionals to tackle climate change: Are China's medical students ready? Environmental Research, 168, 270-277.

Liu, T., Ren, Z., Zhang, Y., Feng, B., Lin, H., Xiao, J., ... \& Xu, Y. (2019). Modification effects of population expansion, ageing, and adaptation on heat-related mortality risks under different climate change scenarios in Guangzhou, China. International Journal of Environmental Research and Public Health, 16(3), 376.

Ma, R., Zhong, S., Morabito, M., Hajat, S., Xu, Z., He, Y., ... \& Huang, C. (2019). Estimation of work-related injury and economic burden attributable to heat stress in Guangzhou, China. Science of the Total Environment, 666, 147-154.

Mazdiyasni, O., AghaKouchak, A., Davis, S. J., Madadgar, S., Mehran, A., Ragno, E., ... \& Niknejad, M. (2017). Increasing probability of mortality during Indian heat waves. Science Advances, 3(6), e1700066.

Phung, D., Chu, C., Tran, D. N., \& Huang, C. (2018). Spatial variation of heat-related morbidity: A hierarchical Bayesian analysis in multiple districts of the Mekong Delta Region. Science of the Total Environment, 637, 1559-1565.

Sheng, R., Li, C., Wang, Q., Yang, L., Bao, J., Wang, K., ... \& Bi, P. (2018). Does hot weather affect work-related injury? A case-crossover study in Guangzhou, China. International Journal of Hygiene and Environmental Health, 221(3), 423-428.

Stocker, T. (Ed.). (2014). Climate change 2013: the physical science basis: Working Group I contribution to the Fifth assessment report of the Intergovernmental Panel on Climate Change. Cambridge University Press.

Stone, B., Hess, J. J., \& Frumkin, H. (2010). Urban form and extreme heat events: are sprawling cities more vulnerable to climate change than compact cities?. Environmental Health Perspectives, 118(10), 1425-1428. Tan, J., Zheng, Y., Song, G., Kalkstein, L. S., Kalkstein, A. J., \& Tang, X. (2007). Heat wave impacts on mortality in Shanghai, 1998 and 2003. International Journal of Biometeorology, 51(3), 193-200.

Wang, Q., Liang, Q., Li, C., Ren, M., Lin, S., Knibbs, L. D., ... \& Wang, X. (2019). Interaction of Air Pollutants and Meteorological Factors on Birth Weight in Shenzhen, China. Epidemiology, 30, S57-S66.

Watts, N., Amann, M., Ayeb-Karlsson, S., Chambers, J., Hamilton, I., Lowe, R., ... \& Latifi, A. M. (2018). The Lancet Countdown on health and climate change: from 25 years of inaction to a global transformation for public health (vol 391, pg 581, 2017). The Lancet, 391(10120), 540.

Wellenius, G. A., Eliot, M. N., Bush, K. F., Holt, D., Lincoln, R. A., Smith, A. E., \& Gold, J. (2017). Heatrelated morbidity and mortality in New England: evidence for local policy. Environmental Research, 156, 845-853.

World Health Organization. (2014). Quantitative risk assessment of the effects of climate change on selected causes of death, 2030s and 2050s. World Health Organization, Geneva.

World Meteorological Organization, \& World Health Organization. (2015). Heatwaves and health: guidance on warning-system development. World Meteorological Organization.

Wolf, T., \& McGregor, G. (2013). The development of a heat wave vulnerability index for London, United Kingdom. Weather and Climate Extremes, 1, 59-68. 